\title{
On the Number of Agents in P Colonies
}

\author{
Luděk Cienciala $^{1}$, Lucie Ciencialová ${ }^{1}$, Alica Kelemenová ${ }^{1,2}$ \\ 1 Institute of Computer Science, Silesian University in Opava, Czech Republic \\ 2 Department of Computer Science, Catholic University Ružomberok, Slovakia \\ \{ludek.cienciala, lucie.ciencialova, alica.kelemenova\}@fpf.slu.cz
}

Summary. We continue the investigation of $\mathrm{P}$ colonies introduced in [7], a class of abstract computing devices composed of independent agents, acting and evolving in a shared environment.

We decrease the number of agents needed to computational completeness of $\mathrm{P}$ colonies with one and two objects inside each agent, respectively, owing some special restrictions to the type of programs. We characterize the generative power of the partially blind machine by the generative power of special $\mathrm{P}$ colonies.

\section{Introduction}

P colonies were introduced in paper [7] as formal models of a computing device inspired by membrane systems and by grammar systems called colonies. This model is inspired by structure and functioning of a community of living organisms in a shared environment.

The independent organisms living in a $\mathrm{P}$ colony are called agents. Each agent is represented by a collection of objects embedded in a membrane. The number of objects inside the agent is the same for each one of them. The environment contains several copies of a basic environmental object denoted by $e$. The number of the copies of $e$ is unlimited.

A set of programs is associated with each agent. The program determines the activity of the agent by rules. In every moment all the objects inside of the agent are being evolved (by an evolution rule) or transported (by a communication rule). The third type of the rules used is a checking rule. This type of the rules sets the priority between two rules.

The computation starts in the initial configuration specified in the definition. Using their programs the agents change themselves and by the environment they can affect the behavior of the other agents. In each step of the computation, each agent with at least one applicable program nondeterministically chooses one of them and executes it. The computation halts when no agent can apply any of its 
programs. The result of the computation is the number of some specific objects present at the environment at the end of the computation.

There are several different ways how to define the beginning of the computation. (1) At the beginning of computation the environment and all agents contain only copies of object $e$. (2) All the agents can contain various objects at the beginning of computation - the agents are in different initial states.

(3) The initial state of the environment is nonempty - the environment contains initial "parameters" for future computation, the agents start with $e$-s.

In $[4,6,7]$ the authors study $\mathrm{P}$ colonies with two objects inside the agents. In this case programs consist of two rules, one for each object. If the former of these rules is an evolution and the latter is a communication or checking, we talk about restricted $\mathrm{P}$ colonies. If we allow also another combination of the types of the rules, we obtain non-restricted $\mathrm{P}$ colonies. The restricted $\mathrm{P}$ colonies with the checking rules are computationally complete [3, 4]. Activities carried out in the field of membrane computing are currently numerous and they are available also at [11].

In the present paper we start with definitions in Section 2.

In Section 3 we will deal with P colonies with one object inside each agent. In recent paper [1] there was shown, that at most seven programs for each agent as well as five agents guarantees the computational completeness of these $\mathrm{P}$ colonies. In the preset paper we look for the generative power of $\mathrm{P}$ colonies with less than five agents. Two results are achieved in this direction. First, we show, that four agents are enough for computational completeness of $\mathrm{P}$ colonies. The second result gives a lower bound for the generative power the $\mathrm{P}$ colonies with two agents. Even a restricted variant of these $\mathrm{P}$ colonies is at least as powerful as the partially blind register machines.

Restricted P colonies are studied in Section 4. It is known that one agent is sufficient to obtain computational completeness of restricted $\mathrm{P}$ systems with checking rules. If no checking rules are used in the restricted $\mathrm{P}$ colonies then we need two agents to prove the universal computational power of those $\mathrm{P}$ colonies.

\section{Definitions}

Throughout the paper we assume the reader to be familiar with the basics of the formal language theory.

We use NRE to denote the family of the recursively enumerable sets of natural numbers. Let $\Sigma$ be the alphabet. Let $\Sigma^{*}$ be the set of all words over $\Sigma$ (including the empty word $\varepsilon$ ). We denote the length of the word $w \in \Sigma^{*}$ by $|w|$ and the number of occurrences of the symbol $a \in \Sigma$ in $w$ by $|w|_{a}$.

A multiset of objects $M$ is a pair $M=(V, f)$, where $V$ is an arbitrary (not necessarily finite) set of objects and $f$ is a mapping $f: V \rightarrow N ; f$ assigns to each object in $V$ its multiplicity in $M$. The set of all multisets with the set of objects $V$ is denoted by $V^{\circ}$. The set $V^{\prime}$ is called the support of $M$ and denoted by $\operatorname{supp}(M)$ if for all $x \in V^{\prime} f(x) \neq 0$ holds. The cardinality of $M$, denoted by 
$|M|$, is defined by $|M|=\sum_{a \in V} f(a)$. Any multiset of objects $M$ with the set of objects $V^{\prime}=\left\{a_{1}, \ldots a_{n}\right\}$ can be represented as a string $w$ over alphabet $V^{\prime}$ with $|w|_{a_{i}}=f\left(a_{i}\right) ; 1 \leq i \leq n$. Obviously, all words obtained from $w$ by permuting the letters can also represent the same $M$, and $\varepsilon$ represents the empty multiset.

\section{1 $\mathrm{P}$ colonies}

We briefly recall the notion of $\mathrm{P}$ colonies. A $\mathrm{P}$ colony consists of agents and environment. Both the agents and the environment contain objects. With every agent the set of program is associated. There are two types of rules in the programs. The first type, called the evolution, is of the form $a \rightarrow b$. It means that object $a$ inside of the agent is rewritten (evolved) to the object $b$. The second type of rules, called a communication, is in the form $c \leftrightarrow d$. When this rule is performed, the object $c$ inside the agent and the object $d$ outside of the agent change their places, so, after execution of the rule $d$ appears inside the agent and $c$ is placed outside of the agent.

In [6] the ability of agents is extended by checking rules. These rules give to the agents an opportunity to opt between two possibilities. They have form $r_{1} / r_{2}$. If the checking rule is performed, the rule $r_{1}$ has higher priority to be executed as the rule $r_{2}$ has. It means that the agent checks the possibility to use rule $r_{1}$. If it can be executed, the agent has to use it. If the first rule cannot be applied, the agent uses the second one.

Definition 1. The $P$ colony of the capacity $k$ is a construct

$$
\Pi=\left(A, e, f, V_{E}, B_{1}, \ldots, B_{n}\right) \text {, where }
$$

- $A$ is an alphabet of the colony, its elements are called objects,

- $e \in A$ is the basic object of the colony,

- $f \in A$ is the final object of the colony,

- $V_{E}$ is a multiset over $A-\{e\}$,

- $B_{i}, 1 \leq i \leq n$, are agents, each agent is a construct $B_{i}=\left(O_{i}, P_{i}\right)$, where

- $O_{i}$ is a multiset over $A$, it determines the initial state (content) of the agent, $\left|O_{i}\right|=k$,

- $P_{i}=\left\{p_{i, 1}, \ldots, p_{i, k_{i}}\right\}$ is a finite multiset of programs, where each program contains exactly $k$ rules, which are in one of the following forms each:

- $a \rightarrow b$, called an evolution rule,

- $c \leftrightarrow d$, called a communication rule,

- $r_{1} / r_{2}$, called a checking rule; $r_{1}, r_{2}$ are an evolution or a communication rules.

An initial configuration of the $\mathrm{P}$ colony is an $(n+1)$-tuple of strings of objects present in the $\mathrm{P}$ colony at the beginning of the computation, it is given by $O_{i}$ for $1 \leq i \leq n$ and by $V_{E}$. Formally, the configuration of $\mathrm{P}$ colony $\Pi$ is given by $\left(w_{1}, \ldots, w_{n}, w_{E}\right)$, where $\left|w_{i}\right|=k, 1 \leq i \leq n, w_{i}$ represents all the objects placed inside the $i$-th agent and $w_{E} \in(A-\{e\})^{*}$ represents all the objects in the environment different from the object $e$. 
In the paper parallel model of $\mathrm{P}$ colonies will be studied. At each step of the parallel computation each agent tries to find one program to use. If the number of applicable programs is higher than one, the agent nondeterministically chooses one of them. At one step of computation the maximal possible number of agents are active.

Let the programs of each $P_{i}$ be labeled in a one-to-one manner by labels in a set $l a b\left(P_{i}\right)$ in such a way that $l a b\left(P_{i}\right) \cap l a b\left(P_{j}\right)=\emptyset$ for $i \neq j, 1 \leq i, j \leq n$.

To express derivation step formally we introduce following four functions for the agent using the rule $r$ of program $p \in P$ with objects $w$ in the environment:

For rule $r$ being $a \rightarrow b, c \leftrightarrow d$ and $c \leftrightarrow d / c^{\prime} \leftrightarrow d^{\prime}$, respectively, and for multiset $w \in V^{\circ}$ we define:

$$
\begin{aligned}
& \text { left }(a \rightarrow b, w)=a \quad \text { left }(c \leftrightarrow d, w)=\varepsilon \\
& \operatorname{right}(a \rightarrow b, w)=b \quad \operatorname{right}(c \leftrightarrow d, w)=\varepsilon \\
& \text { export }(a \rightarrow b, w)=\varepsilon \quad \text { export }(c \leftrightarrow d, w)=c \\
& \text { import }(a \rightarrow b, w)=\varepsilon \quad \text { import }(c \leftrightarrow d, w)=d \\
& \text { left }\left(c \leftrightarrow d / c^{\prime} \leftrightarrow d^{\prime}, w\right)=\varepsilon \\
& \operatorname{right}\left(c \leftrightarrow d / c^{\prime} \leftrightarrow d^{\prime}, w\right)=\varepsilon \\
& \left.\begin{array}{l}
\text { export }\left(c \leftrightarrow d / c^{\prime} \leftrightarrow d^{\prime}, w\right)=c \\
\operatorname{import}\left(c \leftrightarrow d / c^{\prime} \leftrightarrow d^{\prime}, w\right)=d
\end{array}\right\} \text { for }|w|_{d} \geq 1 \\
& \left.\begin{array}{l}
\operatorname{export}\left(c \leftrightarrow d / c^{\prime} \leftrightarrow d^{\prime}, w\right)=c^{\prime} \\
\operatorname{import}\left(c \leftrightarrow d / c^{\prime} \leftrightarrow d^{\prime}, w\right)=d^{\prime}
\end{array}\right\} \text { for }|w|_{d}=0 \text { and }|w|_{d^{\prime}} \geq 1
\end{aligned}
$$

For a program $p$ and any $\alpha \in\{$ left,right, export, import $\}$, let

$$
\alpha(p, w)=\cup_{r \in p} \alpha(r, w) .
$$

A transition from a configuration to another is denoted as are satisfied:

$$
\left(w_{1}, \ldots, w_{n} ; w_{E}\right) \Rightarrow\left(w_{1}^{\prime}, \ldots, w_{n}^{\prime} ; w_{E}^{\prime}\right) \text {, where the following conditions }
$$

- There is a set of program labels $P$ with $|P| \leq n$ such that

- $p, p^{\prime} \in P, p \neq p^{\prime}, p \in l a b\left(P_{j}\right)$ implies $p^{\prime} \notin l a b\left(P_{j}\right)$,

- for each $p \in P, p \in \operatorname{lab}\left(P_{j}\right)$, left $\left(p, w_{E}\right) \cup \operatorname{export}\left(p, w_{E}\right)=w_{j}$, and $\bigcup_{p \in P} \operatorname{import}\left(p, w_{E}\right) \subseteq w_{E}$.

- Furthermore, the chosen set $P$ is maximal, that is, if any other program $r \in \cup_{1 \leq i \leq n} l a b\left(P_{i}\right), r \notin P$, is added to $P$, then the conditions above are not satisfied.

Now, for each $j, 1 \leq j \leq n$, for which there exists a $p \in P$ with $p \in l a b\left(P_{j}\right)$, let $w_{j}^{\prime}=\operatorname{right}\left(p, w_{E}\right) \cup \operatorname{import}\left(p, w_{E}\right)$. If there is no $p \in P$ with $p \in \operatorname{lab}\left(P_{j}\right)$ for some $j, 1 \leq j \leq n$, then let $w_{j}^{\prime}=w_{j}$ and moreover, let

$$
w_{E}^{\prime}=w_{E}-\bigcup_{p \in P}^{i m p o r t}\left(p, w_{E}\right) \cup \bigcup_{p \in P} \operatorname{export}\left(p, w_{E}\right) \text {. }
$$

A configuration is halting if the set of program labels $P$ satisfying the conditions above cannot be chosen to be other than the empty set. A set of all possible halting 
configurations is denoted by $H$. With a halting computation we can associate a result of the computation. It is given by the number of copies of the special symbol $f$ present in the environment. The set of numbers computed by a $\mathrm{P}$ colony $\Pi$ is defined as

$$
N(\Pi)=\left\{\left|v_{E}\right|_{f} \mid\left(w_{1}, \ldots, w_{n}, V_{E}\right) \Rightarrow^{*}\left(v_{1}, \ldots, v_{n}, v_{E}\right) \in H\right\},
$$

where $\left(w_{1}, \ldots, w_{n}, V_{E}\right)$ is the initial configuration, $\left(v_{1}, \ldots, v_{n}, v_{E}\right)$ is a halting configuration, and $\Rightarrow^{*}$ denotes the reflexive and transitive closure of $\Rightarrow$.

Given a $\mathrm{P}$ colony $\Pi=\left(A, e, f, V_{E}, B_{1}, \ldots, B_{n}\right)$ the maximal number of programs associated with the agents in $\mathrm{P}$ colony $\Pi$ is called the height of $\mathrm{P}$ colony $\Pi$. The degree of $\mathrm{P}$ colony $\Pi$ is the number of agents in $\mathrm{P}$ colony $\Pi$. The third parameter characterizing a $\mathrm{P}$ colony is the capacity of $\mathrm{P}$ colony $\Pi$ describing the number of the objects inside each agent.

Let us use the following notations:

$N P C O L_{p a r}(k, n, h)$ for the family of all sets of numbers computed by $\mathrm{P}$ colonies working in parallel, using no checking rules and with:

- the capacity at most $k$,

- the degree at most $n$ and

- the height at most $h$.

If we allow checking rules the family of all sets of numbers computed by $\mathrm{P}$ colonies is denoted by $N P C O L_{p a r} K$. If the $\mathrm{P}$ colonies are restricted, we replace the denotation to $N P C O L_{p a r} R$ and $N P C O L_{\text {par }} K R$, respectively.

\subsection{Register machines}

In this paper we want to characterize the size of the families $N P C O L_{p a r}(k, n, h)$ comparing them with the recursively enumerable sets of numbers. To achieve this aim we use the notion of a register machine.

Definition 2. [8] A register machine is the construct $M=\left(m, H, l_{0}, l_{h}, P\right)$ where:

- $m$ is the number of registers,

- $H$ is the set of instruction labels,

- $l_{0}$ is the start label, $l_{h}$ is the final label,

- $P$ is a finite set of instructions injectively labeled with the elements from the set $H$.

The instruction of the register machine are of the following forms:

$l_{1}:\left(A D D(r), l_{2}, l_{3}\right)$ Add 1 to the content of the register $r$ and proceed to the instruction (labeled with) $l_{2}$ or $l_{3}$.

$l_{1}:\left(S U B(r), l_{2}, l_{3}\right)$ If the register $r$ stores the value different from zero, then subtract 1 from its content and go to instruction $l_{2}$, otherwise proceed to instruction $l_{3}$.

$l_{h}: H A L T \quad$ Stop the machine. The final label $l_{h}$ is only assigned to this instruction.

Without loss of generality, one can assume that in each $A D D$-instruction $l_{1}$ : $\left(A D D(r), l_{2}, l_{3}\right)$ and in each conditional $S U B$-instruction $l_{1}:\left(S U B(r), l_{2}, l_{3}\right)$ the labels $l_{1}, l_{2}, l_{3}$ are mutually distinct. 
The register machine $M$ computes a set $N(M)$ of numbers in the following way: it starts with all registers empty (hence storing the number zero) with the instruction labeled $l_{0}$ and it proceeds to apply the instructions as indicated by the labels (and made possible by the contents of registers). If it reaches the halt instruction, then the number stored at that time in the register 1 is said to be computed by $M$ and hence it is introduced in $N(M)$. (Because of the nondeterminism in choosing the continuation of the computation in the case of $A D D$-instructions, $N(M)$ can be an infinite set.) It is known (see e.g.[8]) that in this way we can compute all sets of numbers which are Turing computable.

Moreover, we call a register machine partially blind [5], if we interpret a subtract instruction in the following way: $l_{1}:\left(S U B(r) ; l_{2} ; l_{3}\right)$ - if in register $r$ there is value different from zero, then subtract one from its contents and go to instruction $l_{2}$ or to instruction $l_{3}$; if in register $r$ there is stored zero when attempting to decrement register $r$, then the program ends without yielding a result.

When the register machine reaches the final state, the result obtained in the first register is only taken into account if the remaining registers store value zero. The family of sets of non-negative integers generated by partially blind register machines is denoted by $N R M_{p b}$. The partially blind register machine accepts a proper subset of NRE.

\section{$3 \mathrm{P}$ colonies with one object inside the agent}

In this Section we analyze the behavior of $\mathrm{P}$ colonies with only one object inside each agent of $\mathrm{P}$ colonies. This gives that every program is formed by only one rule, either an evolution or a communication.

If all the agents have their programs with evolution rules, the agents "live only for themselves" and do not communicate with the environment.

In [1] following results was proved:

$-N P C O L_{p a r} K(1, *, 7)=N R E$.

$-N P C O L_{\text {par }} K(1,5, *)=N R E$

The number of agents in the second result can be decreased:

Theorem 1. $N P C O L_{p a r} K(1,4, *)=N R E$

Proof. We construct a $\mathrm{P}$ colony simulating the computation of the register machine. Because there are only copies of $e$ in the environment and inside the agents, we have to initialize a computation by generating initial label $l_{0}$. After generating symbol $l_{0}$ this agent stops and it can start its activity only by using a program with communicating rule. Two agents will cooperate in order to simulate the ADD and SUB instructions.

Let us consider an $m$-register machine $M=\left(m, H, l_{0}, l_{h}, P\right)$ and present the content of the register $i$ by the number of copies of a specific object $a_{i}$ in the environment. We construct the $\mathrm{P}$ colony $\Pi=\left(A, e, f, \emptyset, B_{1}, \ldots, B_{4}\right)$ with: 


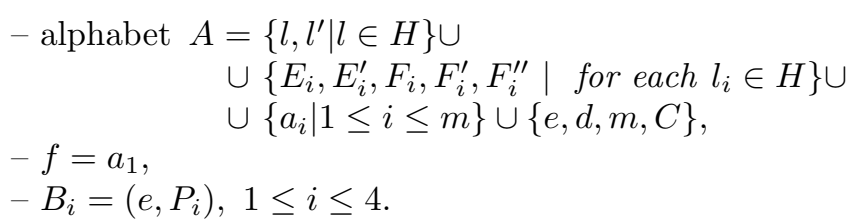

(1) To initialize simulation of computation of $M$ we take agent $B_{1}=\left(e, P_{1}\right)$ with a set of programs:

$$
\frac{P_{1}:}{1:\left\langle e \rightarrow l_{0}\right\rangle, 2:\left\langle l_{0} \leftrightarrow d\right\rangle ;}
$$

(2) We need one more agent to generate some special object $d$. In every pair of steps the agent $B_{2}$ places one copy of $d$ to the environment.

$$
\frac{P_{2}:}{3:\langle e \rightarrow d\rangle, 4:\langle d \leftrightarrow C / d \leftrightarrow e\rangle ;}
$$

The $\mathrm{P}$ colony $\Pi$ starts its computation in the initial configuration $(e, e, e, e, \varepsilon)$. In the first subsequence of steps of $\mathrm{P}$ colony $\Pi$ only agents $B_{1}, B_{2}$ can apply its

\begin{tabular}{|c|c|c|c|c|c|c|c|c|c|}
\hline \multirow[b]{2}{*}{ step } & \multicolumn{5}{|c|}{ configuration of $\Pi$} & \multirow[b]{2}{*}{$P_{1}$} & \multirow[b]{2}{*}{$P_{2}$} & \multirow[b]{2}{*}{$P_{3}$} & \multirow[b]{2}{*}{$P_{4}$} \\
\hline & $B_{1}$ & $B_{2}$ & $B_{3}$ & $B_{4}$ & Env & & & & \\
\hline 1. & $e$ & $e$ & $e$ & $e$ & & 1 & 3 & & \\
\hline 2. & $l_{0}$ & $d$ & $e$ & $e$ & & & 4 & & \\
\hline 3. & $l_{0}$ & $e$ & $e$ & $e$ & $d$ & 2 & 3 & & \\
\hline 4. & $d$ & $d$ & $e$ & $e$ & $l_{0}$ & & & & \\
\hline
\end{tabular}
programs.

(3) To simulate the ADD-instruction $l_{1}:\left(A D D(r), l_{2}, l_{3}\right)$ there are two agents $B_{3}$ and $B_{4}$ in $\mathrm{P}$ colony $\Pi$. These agents help each other to add one copy of object $a_{r}$ and object $l_{2}$ or $l_{3}$ to the environment.

\begin{tabular}{llll}
$P_{3}$ & $P_{3}$ & $P_{4}$ & $P_{4}$ \\
\hline $5:\left\langle e \leftrightarrow l_{1}\right\rangle$, & $11:\left\langle E_{1}^{\prime} \rightarrow l_{2}^{\prime}\right\rangle$, & $15:\left\langle e \leftrightarrow E_{1}\right\rangle$, & $21:\left\langle e \leftrightarrow l_{2}^{\prime}\right\rangle$, \\
$6:\left\langle l_{1} \rightarrow E_{1}\right\rangle$, & $12:\left\langle E_{1}^{\prime} \rightarrow l_{3}^{\prime}\right\rangle$, & $16:\left\langle E_{1} \rightarrow E_{1}^{\prime}\right\rangle$, & $22:\left\langle e \leftrightarrow l_{3}^{\prime}\right\rangle$, \\
$7:\left\langle E_{1} \leftrightarrow d\right\rangle$, & $13:\left\langle l_{2}^{\prime} \leftrightarrow e\right\rangle$, & $17:\left\langle E_{1}^{\prime} \leftrightarrow e\right\rangle$, & $23:\left\langle l_{2}^{\prime} \rightarrow l_{2}\right\rangle$, \\
$8:\left\langle d \rightarrow L_{1}\right\rangle$, & $14:\left\langle l_{3}^{\prime} \leftrightarrow e\right\rangle$, & $18:\left\langle e \leftrightarrow L_{1}\right\rangle$, & $24:\left\langle l_{3}^{\prime} \rightarrow l_{3}\right\rangle$, \\
$9:\left\langle L_{1} \leftrightarrow E_{1}^{\prime} / L_{1} \rightarrow m\right\rangle$, & $19:\left\langle L_{1} \leftarrow a_{r}\right\rangle$, & $25:\left\langle l_{2} \leftrightarrow e\right\rangle$, \\
$10:\langle m \rightarrow d\rangle$, & $20:\left\langle a_{r} \leftrightarrow e\right\rangle$, & $26:\left\langle l_{3} \leftrightarrow e\right\rangle ;$
\end{tabular}

The agent $B_{3}$ consumes the object $l_{1}$, changes it to $E_{1}$ and places it to the environment. The agent $B_{4}$ borrows $E_{1}$ from the environment and gives a little altered (to $E_{1}^{\prime}$ ) back. $B_{3}$ rewrites the object $d$ to some $L_{i}$. If this $L_{i}$ has the same index as $E_{i}^{\prime}$ placed in the environment, the computation can go to the next phase. If indices of $L_{i}$ and $E_{i}$ are different the agent $B_{3}$ tries to generate another $L_{i}$. If the computation gets over this checking step, $B_{3}$ generates the helpful object $l_{2}^{\prime}$ or $l_{3}^{\prime}$ and places it to the environment. The agent $B_{4}$ exchanges it for "valid label" $l_{2}$ or $l_{3}$.

An instruction $l_{i}:\left(A D D(r), l_{j}, l_{k}\right)$ is simulated by the following sequence of steps. Let the content of the agent $B_{2}$ be $d$. 


\begin{tabular}{|c|c|c|c|c|c|c|c|c|c|}
\hline \multirow[b]{2}{*}{ step } & \multicolumn{5}{|c|}{ configuration of $\Pi$} & \multirow[b]{2}{*}{$P_{1}$} & \multirow[b]{2}{*}{$P_{2}$} & \multirow[b]{2}{*}{$P_{3}$} & \multirow[b]{2}{*}{$P_{4}$} \\
\hline & $B_{1}$ & $B_{2}$ & $B_{3}$ & $B_{4}$ & Env & & & & \\
\hline 1. & $d$ & $d$ & $e$ & $e$ & $l_{i} a_{r}^{u} d^{v}$ & & 4 & 5 & \multirow[b]{4}{*}{5} \\
\hline 2. & $d$ & $e$ & $l_{i}$ & $e$ & $a_{r}^{u} d^{v+1}$ & & 3 & 6 & \\
\hline 3. & $d$ & $d$ & $E_{i}$ & $e$ & $a_{r}^{u} d^{v+1} d$ & & 4 & 7 & \\
\hline 4. & $d$ & $e$ & $d$ & $e$ & $E_{i} a_{r}^{u} d^{v+1}$ & & 3 & 8 & \\
\hline & \multicolumn{5}{|c|}{ configuration of $\Pi$} & \multirow[b]{2}{*}{$P_{1}$} & & \multirow[b]{2}{*}{$P_{3}$} & \multirow[b]{2}{*}{$P_{4}$} \\
\hline step & $B_{1}$ & $B_{2}$ & $B_{3}$ & $B_{4}$ & Env & & $P_{2}$ & & \\
\hline 5. & $d$ & $d$ & $L_{i}$ & $E_{i}$ & $a_{r}^{u} d^{v+1}$ & & 4 & \multirow[b]{3}{*}{9} & 16 \\
\hline 6. & $d$ & $e$ & $L_{i}$ & $E_{i}^{\prime}$ & $a_{r}^{u} d^{v+2}$ & & 3 & & 17 \\
\hline 7. & $d$ & $d$ & $L_{i}$ & $e$ & $E_{i}^{\prime} a_{r}^{u} d^{v+2}$ & & 4 & & \\
\hline 8. & $d$ & $e$ & $E_{i}^{\prime}$ & $e$ & $L_{i} a_{r}^{u} d^{v+3}$ & & 3 & 11 or 12 & 18 \\
\hline 9. & $d$ & $d$ & $l_{j}^{\prime}$ & $L_{i}$ & $a_{r}^{u} d^{v+3}$ & & 4 & 13 & 19 \\
\hline 10 . & $d$ & $e$ & $e$ & $a_{r}$ & $l_{j}^{\prime} a_{r}^{u} d^{v+4}$ & & 3 & & 20 \\
\hline 11. & $d$ & $d$ & $e$ & $e$ & $l_{j}^{\prime} a_{r}^{u+1} d^{v+4}$ & & 4 & & 21 \\
\hline 12. & $d$ & $e$ & $e$ & $l_{j}^{\prime}$ & $a_{r}^{u+1} d^{v+5}$ & & 3 & & 23 \\
\hline 13. & $d$ & $d$ & $e$ & $l_{j}$ & $a_{r}^{u+1} d^{v+5}$ & & 4 & & 25 \\
\hline 14 . & $d$ & $e$ & $e$ & $e$ & $l_{j} a_{r}^{u+1} d^{v+6}$ & & & & \\
\hline
\end{tabular}

(4) For each SUB-instruction $l_{1}:\left(S U B(r), l_{2}, l_{3}\right)$, the next programs are introduced in the sets $P_{1}, P_{3}$ and in the set $P_{4}$ :

\begin{tabular}{llll}
$P_{3}$ & $P_{3}$ & $P_{1}$ & $P_{4}$ \\
\hline $27:\left\langle e \leftrightarrow l_{1}\right\rangle$, & $33:\left\langle F_{1}^{\prime \prime} \rightarrow l_{3}^{\prime}\right\rangle$, & $36:\left\langle d \leftrightarrow F_{1}\right\rangle$, & $41:\left\langle e \leftrightarrow l_{2}^{\prime}\right\rangle$, \\
$28:\left\langle l_{1} \rightarrow F_{1}\right\rangle$, & $34:\left\langle l_{2}^{\prime} \leftrightarrow e\right\rangle$, & $37:\left\langle F_{1} \rightarrow F_{1}^{\prime}\right\rangle$, & $42:\left\langle e \leftrightarrow l_{3}^{\prime}\right\rangle$, \\
$29:\left\langle F_{1} \leftrightarrow d\right\rangle$, & $35:\left\langle l_{3}^{\prime} \leftrightarrow e\right\rangle ;$ & $38:\left\langle F_{1}^{\prime} \leftrightarrow a_{r} / F_{1}^{\prime} \rightarrow F_{1}^{\prime \prime}\right\rangle$, & $43:\left\langle l_{2}^{\prime} \rightarrow l_{2}\right\rangle$, \\
$30:\left\langle d \leftrightarrow F_{1}^{\prime}\right\rangle$, & & $39:\left\langle a_{r} \rightarrow d\right\rangle$, & $44:\left\langle l_{3}^{\prime} \rightarrow l_{3}\right\rangle$, \\
$31:\left\langle F_{1}^{\prime} \rightarrow l_{2}^{\prime}\right\rangle$, & $40:\left\langle F_{1}^{\prime \prime} \leftrightarrow d\right\rangle$, & $45:\left\langle l_{2} \leftrightarrow e\right\rangle$, \\
$32:\left\langle d \leftrightarrow F_{1}^{\prime \prime}\right\rangle$, & & $46:\left\langle l_{3} \leftrightarrow e\right\rangle$
\end{tabular}

Agent $B_{4}$ starts simulation of executing SUB-instruction $l_{1}$, the agent $B_{1}$ checks whether there is a copy of the object $a_{r}$ in the environment or not and gives this information ( $F_{1}^{\prime}$ - there is some $a_{r} ; F_{1}^{\prime \prime}$ - there is no object $a_{r}$ in the environment) to the environment.

An instruction $l_{i}:\left(S U B(r), l_{j}, l_{k}\right)$ is simulated by the following sequence of steps. When the value in counter $r$ is zero: 


\begin{tabular}{|c|ccccc|cccc|}
\hline & \multicolumn{7}{|c|}{ configuration of $\Pi$} & \multicolumn{5}{c|}{ applicable programs } \\
step & $B_{1}$ & $B_{2}$ & $B_{3}$ & $B_{4}$ & $E n v$ & $P_{1}$ & $P_{2}$ & $P_{3}$ & $P_{4}$ \\
\hline 1. & $d$ & $d$ & $e$ & $e$ & $l_{i} d^{v}$ & & 4 & 27 & \\
2. & $d$ & $e$ & $l_{i}$ & $e$ & $d^{v+1}$ & 3 & 28 & \\
3. & $d$ & $d$ & $F_{i}$ & $e$ & $d^{v+1} d$ & & 4 & 29 & \\
4. & $d$ & $e$ & $d$ & $e$ & $F_{i} d^{v+1}$ & 36 & 3 & & \\
5. & $F_{i}$ & $d$ & $d$ & $e$ & $d^{v+2}$ & 37 & 4 & & \\
6. & $F_{i}^{\prime}$ & $e$ & $d$ & $e$ & $d^{v+3}$ & 38 & 3 & & \\
7. & $F_{i}^{\prime \prime}$ & $d$ & $d$ & $e$ & $d^{v+3}$ & 40 & 4 & & \\
8. & $d$ & $e$ & $d$ & $e$ & $F_{i}^{\prime \prime} d^{v+3}$ & 3 & 32 & \\
9. & $d$ & $d$ & $F_{i}^{\prime \prime}$ & $e$ & $d^{v+4}$ & & 4 & 33 & \\
10. & $d$ & $e$ & $l_{k}^{\prime}$ & $e$ & $d^{v+5}$ & 3 & 35 & \\
11. & $d$ & $d$ & $e$ & $e$ & $l_{k}^{\prime} d^{v+5}$ & 4 & & 42 \\
12. & $d$ & $e$ & $e$ & $l_{k}^{\prime}$ & $d^{v+6}$ & & 3 & & 44 \\
13. & $d$ & $d$ & $e$ & $l_{k}$ & $d^{v+6}$ & & 4 & & 46 \\
14. & $d$ & $e$ & $e$ & $e$ & $l_{k} d^{v+7}$ & & & \\
\hline
\end{tabular}

When register $r$ stores value different from zero:

\begin{tabular}{|c|ccccc|cccc|}
\hline & \multicolumn{7}{|c|}{ configuration of $\Pi$} \\
step & $B_{1}$ & $B_{2}$ & $B_{3}$ & $B_{4}$ & $E n v$ & $P_{1}$ & $P_{2}$ & $P_{3}$ & $P_{4}$ \\
\hline 1. & $d$ & $d$ & $e$ & $e$ & $l_{i} a_{r}^{u} d^{v}$ & & 27 & \\
2. & $d$ & $e$ & $l_{i}$ & $e$ & $a_{r}^{u} d^{v+1}$ & 3 & 28 & \\
3. & $d$ & $d$ & $F_{i}$ & $e$ & $a_{r}^{u} d^{v+1} d$ & & 4 & 29 & \\
4. & $d$ & $e$ & $d$ & $e$ & $F_{i} a_{r}^{u} d^{v+1}$ & 36 & 3 & & \\
5. & $F_{i}$ & $d$ & $d$ & $e$ & $a_{r}^{u} d^{v+2}$ & 37 & 4 & & \\
6. & $F_{i}^{\prime}$ & $e$ & $d$ & $e$ & $a_{r}^{u} d^{v+3}$ & 38 & 3 & & \\
7. & $a_{r}$ & $d$ & $d$ & $e$ & $F_{i} a_{r}^{u-1} d^{v+3}$ & 39 & 4 & 30 & \\
8. & $d$ & $e$ & $F_{i}^{\prime}$ & $e$ & $a_{r}^{u-1} d^{v+5}$ & & 3 & 31 & \\
9. & $d$ & $d$ & $l_{j}^{\prime}$ & $e$ & $a_{r}^{u-1} d^{v+5}$ & & 3 & 34 & \\
10. & $d$ & $e$ & $e$ & $e$ & $l_{j}^{\prime} a_{r}^{u-1} d^{v+6}$ & 3 & & 41 \\
11. & $d$ & $d$ & $e$ & $l_{j}^{\prime}$ & $a_{r}^{u-1} d^{v+6}$ & & & 4 \\
12. & $d$ & $e$ & $e$ & $l_{j}$ & $a_{r}^{u-1} d^{v+7}$ & & 3 & & 45 \\
13. & $d$ & $d$ & $e$ & $e$ & $l_{j} a_{r}^{u-1} d^{v+7}$ & & & \\
\hline
\end{tabular}

(5) The halting instruction $l_{h}$ is simulated by agent $B_{3}$ with subset of programs:

$$
\frac{P_{3}}{47:\left\langle e \leftrightarrow l_{h}\right\rangle, 48:\left\langle l_{h} \rightarrow C\right\rangle, 49:\langle C \leftrightarrow e\rangle .}
$$

The agent consumes the object $l_{h}$ and in the environment there is no other object $l_{m}$. This agent places one copy of the object $C$ to the environment and stops working. In the next step the object $C$ is consumed by the agent $B_{3}$. No agent can start its work and computation halts. The execution of halting instruction $l_{h}$ stops all agents in $\mathrm{P}$ colony $\Pi$ : 


\begin{tabular}{|c|ccccc|cccc|}
\hline & \multicolumn{7}{|c|}{ configuration of $\Pi$} \\
step & $B_{1}$ & $B_{2}$ & $B_{3}$ & $B_{4}$ & $E n v$ & $P_{1}$ & $P_{2}$ & $P_{3}$ & $P_{4}$ \\
\hline 1. & $d$ & $d$ & $e$ & $e$ & $l_{h} d^{v}$ & 4 & 47 & \\
2. & $d$ & $e$ & $l_{h}$ & $e$ & $d^{v+1}$ & 3 & 48 & \\
3. & $d$ & $d$ & $C$ & $e$ & $d^{v+1} d$ & 4 & 49 & \\
4. & $d$ & $e$ & $e$ & $e$ & $C d^{v+1}$ & 3 & & \\
5. & $d$ & $d$ & $e$ & $e$ & $C d^{v+2}$ & 4 & & \\
6. & $d$ & $C$ & $e$ & $e$ & $d^{v+3}$ & $\ldots \ldots \ldots$ \\
\hline
\end{tabular}

P colony $\Pi$ correctly simulates computation in the register machine $M$. The computation of $\Pi$ starts with no object $a_{r}$ placed in the environment in the same way as the computation in $M$ starts with zeros in all the registers. The computation of $\Pi$ stops if the symbol $l_{h}$ is placed inside the corresponding agent in the same way as $M$ stops by executing the halting instruction labeled $l_{h}$. Consequently, $N(M)=N(\Pi)$ and because the number of agents equals four, the proof is complete.

Theorem 2. $N R M_{p b} \subseteq N P C O L_{p a r}(1,2, *)$.

Proof. Let us consider a partially blind register machine $M$ with $m$ registers. We construct a $\mathrm{P}$ colony $\Pi=\left(A, e, f, V_{E}, B_{1}, B_{2}\right)$ simulating a computation of the register machine $M$ with:

$$
\begin{aligned}
& -A=\left\{J, J^{\prime}, V, Q\right\} \cup\left\{l_{i}, l_{i}^{\prime}, l_{i}^{\prime \prime}, L_{i}, L_{i}^{\prime}, L_{i}^{\prime \prime}, E_{i} \mid l_{i} \in H\right\} \cup\left\{a_{r} \mid 1 \leq r \leq m\right\}, \\
& -f=a_{1}, \\
& \text { - } B_{i}=\left(O_{i}, P_{i}\right), O_{i}=\{e\}, i=1,2
\end{aligned}
$$

The sets of programs are as follows:

(1) For initializing the simulation:

$$
\begin{array}{lll}
P_{1}: & P_{1}: & P_{2}: \\
\hline 1:\langle e \rightarrow J\rangle, & 3:\left\langle J \rightarrow l_{0}\right\rangle, & 5:\langle e \leftrightarrow J\rangle, \\
2:\langle J \leftrightarrow e\rangle, & 4:\langle Q \rightarrow Q\rangle, & 6:\left\langle J \rightarrow J^{\prime}\right\rangle, \\
& & 7:\left\langle J^{\prime} \leftrightarrow e\right\rangle ;
\end{array}
$$

At the beginning of the computation the first agent generates the object $l_{0}$ (the label of starting instruction of $M$ ). It generates some copies of object $J$. The agent $B_{2}$ exchange them by $J^{\prime}$.

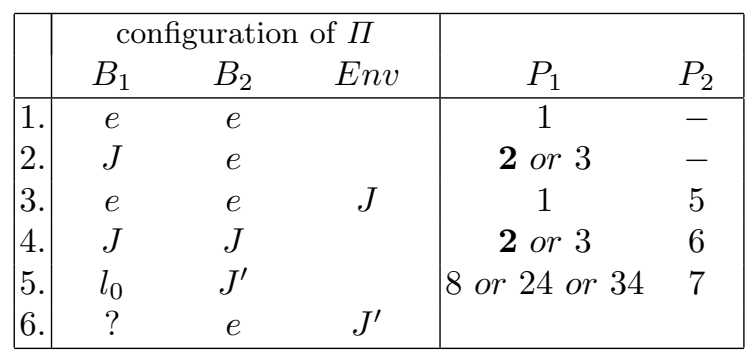

(2) For every $A D D$-instruction $l_{1}:\left(A D D(r), l_{2}, l_{3}\right) P_{1}$ and $P_{2}$ contain: 


\begin{tabular}{cll}
$P_{1}:$ & $P_{1}:$ & $P_{2}:$ \\
\hline $8:\left\langle l_{1} \rightarrow l_{1}^{\prime}\right\rangle$, & $14:\left\langle L_{1} \leftrightarrow E_{1}\right\rangle$, & $18:\left\langle e \leftrightarrow l_{1}^{\prime}\right\rangle$, \\
$9:\left\langle l_{1}^{\prime} \leftrightarrow J^{\prime}\right\rangle$, & $15:\left\langle L_{1} \rightarrow Q\right\rangle$, & $19:\left\langle l_{1}^{\prime} \rightarrow E_{1}\right\rangle$, \\
$10:\left\langle l_{1}^{\prime} \rightarrow Q\right\rangle$, & $16:\left\langle E_{1} \rightarrow l_{2}\right\rangle$ & $20:\left\langle E_{1} \leftrightarrow e\right\rangle$, \\
$11:\left\langle J^{\prime} \rightarrow L_{1}^{\prime \prime}\right\rangle$, & $17:\left\langle E_{1} \rightarrow l_{3}\right\rangle$ & $21:\left\langle e \leftrightarrow L_{1}\right\rangle$ \\
$12:\left\langle L_{1}^{\prime \prime} \rightarrow L_{1}^{\prime}\right\rangle$, & & $22:\left\langle L_{1} \rightarrow a_{r}\right\rangle$ \\
$13:\left\langle L_{1}^{\prime} \rightarrow L_{1}\right\rangle$, & & $23:\left\langle a_{r} \leftrightarrow e\right\rangle$
\end{tabular}

When there is object $l_{1}$ inside agent $B_{1}$, the agent rewrites it to one copy of $l_{1}^{\prime}$ and the agent sends it to the environment. The agent $B_{2}$ borrows $E_{1}$ from the environment and returns $E_{1}^{\prime}$ back.

The agent $B_{1}$ rewrites the object $J^{\prime}$ to some $L_{i}$. The first agent has to generate it in three steps to wait till the second agent generates the symbol $E_{i}^{\prime}$ and places it to the environment. If this $L_{i}$ has the same index as $E_{i}^{\prime}$ placed in the environment, the computation can go to the next phase. If the indices of $L_{i}$ and $E_{i}$ are different, the agent $B_{1}$ generates $Q$ and the computation never stops. If the computation gets over this checking step, $B_{1}$ generates object $l_{2}$ or $l_{3}$.

\begin{tabular}{|c|ccc|cc|}
\hline & \multicolumn{3}{|c|}{ configuration of $\Pi$} & & \\
& $B_{1}$ & $B_{2}$ & $E n v$ & $P_{1}$ & $P_{2}$ \\
\hline 1. & $l_{1}$ & $e$ & $J^{\prime}$ & 8 & - \\
2. & $l_{1}^{\prime}$ & $e$ & $J^{\prime}$ & $\mathbf{9}$ or 10 & - \\
3. & $J^{\prime}$ & $e$ & $l_{1}^{\prime}$ & 11 & 18 \\
4. & $L_{1}^{\prime \prime}$ & $l_{1}^{\prime}$ & & 12 & 19 \\
5. & $L_{1}^{\prime}$ & $E_{1}$ & & 13 & 20 \\
6. & $L_{1}$ & $e$ & $E_{1}$ & $\mathbf{1 4}$ or 15 & - \\
7. & $E_{1}$ & $e$ & $L_{1}$ & $\mathbf{1 6}$ or 17 & 21 \\
8. & $l_{2}$ & $L_{1}$ & & 8 or 24 or 34 & 22 \\
9. & $?$ & $a_{r}$ & & 9 or 25 or 35 & 23 \\
10. & $?$ & $e$ & $a_{r}$ & & \\
\hline
\end{tabular}

(3) For every $S U B$-instruction $l_{1}:\left(S U B(r), l_{2}, l_{3}\right)$ there are subsets of programs in $P_{1}$ and $P_{2}$ :

\begin{tabular}{lll}
$P_{1}:$ & $P_{1}:$ & $P_{2}:$ \\
\hline $24:\left\langle l_{1} \rightarrow l_{1}^{\prime \prime}\right\rangle$, & $28:\left\langle V \leftrightarrow l_{1}^{\prime \prime \prime}\right\rangle$, & $31:\left\langle l_{1}^{\prime \prime} \leftrightarrow e\right\rangle$, \\
$25:\left\langle l_{1}^{\prime \prime} \leftrightarrow a_{r}\right\rangle$, & $29:\left\langle l_{1}^{\prime \prime \prime} \rightarrow l_{2}\right\rangle$, & $32:\left\langle l_{1}^{\prime \prime} \rightarrow l_{1}^{\prime \prime \prime}\right\rangle$, \\
$26:\left\langle l_{1}^{\prime \prime} \rightarrow Q\right\rangle$, & $30:\left\langle l_{1}^{\prime \prime \prime} \rightarrow l_{3}\right\rangle$ & $33:\left\langle l_{1}^{\prime \prime \prime} \leftrightarrow e\right\rangle$,
\end{tabular}

In the first step the agent checks if there is any copy of $a_{r}$ in the environment (for zero in register $r$ ). In the positive case it rewrites $a_{r}$ to $V$, in the other case $l_{1}^{\prime \prime}$ is rewritten to $Q$ and the computation will never halt. At the end of this simulation the agent $B_{1}$ generates object $l_{2}$ or $l_{3}$. 


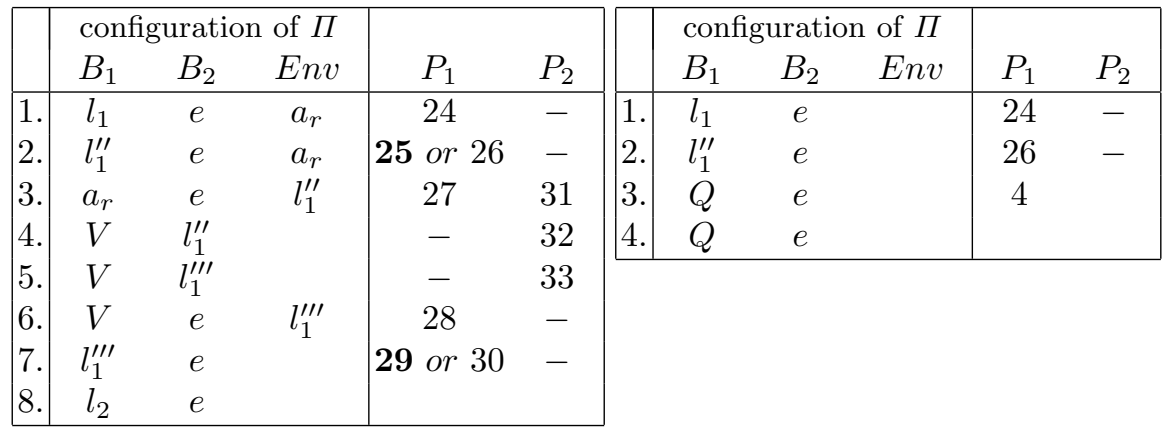

(4) For halting instruction $l_{h}$ there are programs in sets $P_{1}$ and $P_{2}$ :

$$
\begin{array}{lll}
P_{1}: & P_{2}: & P_{2}: \\
\hline 34:\left\langle l_{h} \leftrightarrow J^{\prime}\right\rangle, & 39:\left\langle e \leftrightarrow l_{h}\right\rangle, & 43:\left\langle L_{h} \leftrightarrow a_{r}\right\rangle, 1<r \leq m \\
35:\left\langle J^{\prime} \rightarrow L_{h}\right\rangle, & 40:\left\langle l_{h} \rightarrow \overline{l_{h}}\right\rangle, & 44:\left\langle a_{r} \leftrightarrow e\right\rangle \\
36:\left\langle l_{h} \rightarrow Q\right\rangle, & 41:\left\langle\overline{\left.l_{h} \leftrightarrow e\right\rangle,}\right. & \\
37:\left\langle L_{h} \rightarrow L_{h}\right\rangle, & 42:\left\langle e \leftrightarrow L_{h}\right\rangle & \\
38:\left\langle L_{h} \leftrightarrow \overline{l_{h}}\right\rangle, & &
\end{array}
$$

\begin{tabular}{|c|c|c|c|c|c|c|c|c|c|c|c|}
\hline \multicolumn{6}{|c|}{ all counters $r, 1<r \leq m$ store zero } & \multirow{2}{*}{\multicolumn{6}{|c|}{$\begin{array}{c}\text { content of some counter } r, 1<r \leq m \text { is } \\
\text { different from zero }\end{array}$}} \\
\hline & \multicolumn{3}{|c|}{ configuration of $\Pi$} & \multirow[b]{2}{*}{$P_{1}$} & \multirow[b]{2}{*}{$P_{2}$} & & & & & & \\
\hline & $B_{1}$ & $B_{2}$ & Env & & & & \multicolumn{3}{|c|}{ configuration of $\Pi$} & \multirow[b]{2}{*}{$P_{1}$} & \multirow[b]{2}{*}{$P_{2}$} \\
\hline 1. & $l_{h}$ & $e$ & $J^{\prime}$ & 34 or 36 & - & & $B_{1}$ & $B_{2}$ & Env & & \\
\hline 2 . & $J^{\prime}$ & $e$ & $l_{h}$ & 35 & 39 & 1. & $l_{h}$ & $e$ & $J^{\prime} a_{r}$ & 34 or 36 & - \\
\hline 3. & $L_{h}$ & $l_{h}$ & & 37 & 40 & 2 . & $J^{\prime}$ & $e$ & $l_{h} a_{r}$ & 35 & 39 \\
\hline 4. & $L_{h}$ & $\overline{l l}$ & & 37 & 41 & 3. & $L_{h}$ & $l_{h}$ & $a_{r}$ & 37 & 40 \\
\hline 5. & $L_{H}$ & $e$ & $\overline{l_{h}}$ & 38 & - & 4. & $L_{h}$ & $\overline{l_{h}}$ & $a_{r}$ & 37 & 41 \\
\hline 6. & $\overline{l_{h}}$ & $e$ & $L_{h}$ & - & 42 & 5 . & $L_{H}$ & $e$ & $\overline{l_{h}} a_{r}$ & 38 & - \\
\hline 7. & $\overline{l_{h}}$ & $L_{h}$ & & - & - & 6. & $\overline{l_{h}}$ & $e$ & $L_{h} a_{r}$ & - & 42 \\
\hline & & & & & & 7. & $\overline{l_{h}}$ & $L_{h}$ & $a_{r}$ & - & 43 \\
\hline & & & & & & 8. & $\overline{l_{h}}$ & $a_{r}$ & $L_{h}$ & - & 44 \\
\hline & & & & & & 9. & $\overline{l_{h}}$ & $L_{h}$ & $a_{r}$ & - & 43 \\
\hline
\end{tabular}

By using these programs, the $\mathrm{P}$ colony finishes the computation in the same way as the partially blind register machine halts its computation. Programs with labels 43 and 44 in $P_{2}$ check value zero stored in all except the first one registers.

P colony $\Pi$ correctly simulates any computation of the partially blind register machine $M$.

\section{On computational power of restricted $\mathrm{P}$ colonies without checking}

For restricted $\mathrm{P}$ colonies Following results are known from the literature: 
- $N P C O L_{p a r} K R(2, *, 5)=N R E$ in $[2,7]$,

- $N P C O L_{p a r} R(2, *, 5)=N P C O L_{p a r} K R(2,1, *)=N R E$ in [4].

Theorem 3. $N P C O L_{p a r} R(2,2, *)=N R E$.

Proof. Let us consider a register machine $M$ with $m$ registers. We construct a $\mathrm{P}$ colony $\Pi=\left(A, e, f, V_{e}, B_{1}, B_{2}\right)$ simulating the computations of register machine $M$ with:

$$
\begin{aligned}
& -A=\{G\} \cup\left\{l_{i}, l_{i}^{\prime}, l_{i}^{\prime \prime}, l_{i}^{\prime \prime \prime}, l_{i}^{\prime \prime \prime}, \overline{l_{i}}, \overline{\overline{l_{i}}}, \underline{l_{i}}, \underline{\underline{l_{i}}}, L_{i}, L_{i}^{\prime}, L_{i}^{\prime \prime}, F_{i} \mid l_{i} \in H\right\} \cup \\
& \quad \cup\left\{a_{r} \mid 1 \leq r \leq m\right\}, \\
& -f=a_{1}, \\
& -B_{j}=\left(O_{j}, P_{j}\right), O_{j}=\{e, e\}, j=1,2
\end{aligned}
$$

At the beginning of the computation the first agent generates the object $l_{0}$ (the label of starting instruction of $M$ ). Then it starts to simulate instruction labeled $l_{0}$ and it generates the label of the next instruction. The sets of programs are as follows:

(1) For initializing of the simulation there is one program in $P_{1}$ :

\section{$\frac{P_{1}}{1:\left\langle e \rightarrow l_{0} ; e \leftrightarrow e\right\rangle}$}

The initial configuration of $\Pi$ is $(e e, e e, \varepsilon)$. After the first step of computation (only the program 1 is applicable) the system enters configuration $\left(l_{0} e, e e, \varepsilon\right)$.

(2) For every $A D D$-instruction $l_{1}:\left(A D D(r), l_{2}, l_{3}\right)$ we add to $P_{1}$ the programs:

$$
\begin{array}{ll}
P_{1} & \\
\hline 2:\left\langle e \rightarrow a_{r} ; l_{1} \leftrightarrow e\right\rangle, & 3:\left\langle e \rightarrow G ; a_{r} \leftrightarrow l_{1}\right\rangle, \\
4:\left\langle l_{1} \rightarrow l_{2} ; G \leftrightarrow e\right\rangle, & 5:\left\langle l_{1} \rightarrow l_{3} ; G \leftrightarrow e\right\rangle
\end{array}
$$

When there is object $l_{1}$ inside the agent, it generates one copy of $a_{r}$, puts it to the environment and generates the label of the next instruction (it nondeterministically chooses one of the last two programs 4 and 5)

\begin{tabular}{|c|ccc|cc|}
\hline & \multicolumn{6}{|c|}{ configuration of $\Pi$} & & \\
& $B_{1}$ & $B_{2}$ & $E n v$ & $P_{1}$ & $P_{2}$ \\
\hline 1. & $l_{1} e$ & $e e$ & $a_{r}^{x}$ & 2 & - \\
2. & $a_{r} e$ & $e e$ & $l_{1} a_{r}^{x}$ & 3 & - \\
3. & $G l_{1}$ & $e e$ & $a_{r}^{x+1}$ & 4 or 5 & - \\
4. & $l_{2} e$ & $e e$ & $a_{r}^{x+1} G$ & & \\
\hline
\end{tabular}

(3) For every $S U B$-instruction $l_{1}:\left(S U B(r), l_{2}, l_{3}\right)$, the next programs are added to sets $P_{1}$ and $P_{2}$ : 


\begin{tabular}{|c|c|c|}
\hline$P_{1}$ & $P_{1}$ & $P_{2}$ \\
\hline $6:\left\langle l_{1} \rightarrow l_{1}^{\prime} ; e \leftrightarrow e\right\rangle$ & $12:\left\langle\overline{\overline{l_{1}}} \rightarrow \underline{l_{2}} ; e \leftrightarrow L_{1}^{\prime \prime}\right\rangle$ & $\overline{18:\left\langle e \rightarrow L_{1} ; e \leftrightarrow l_{1}^{\prime}\right\rangle}$ \\
\hline $7:\left\langle e \rightarrow l_{1}^{\prime \prime} ; l_{1}^{\prime} \leftrightarrow e\right\rangle$ & $13:\left\langle\overline{\overline{l_{1}}} \rightarrow \underline{l_{3}} ; e \leftrightarrow L_{1}\right\rangle$ & $19:\left\langle l_{1}^{\prime} \rightarrow L_{1}^{\prime} ; L_{1} \leftrightarrow l_{1}^{\prime \prime}\right\rangle$ \\
\hline $8:\left\langle e \rightarrow l_{1}^{\prime \prime \prime} ; l_{1}^{\prime \prime} \leftrightarrow e\right\rangle$ & $14:\left\langle L_{1}^{\prime \prime} \rightarrow l_{2} ; l_{2} \leftrightarrow e\right\rangle$ & $20:\left\langle l_{1}^{\prime \prime} \rightarrow L_{1}^{\prime \prime} ; L_{1}^{\prime} \leftrightarrow a_{r}\right\rangle$ \\
\hline $9:\left\langle l_{1}^{\prime \prime \prime} \rightarrow l_{1}^{\prime \prime \prime \prime} ; e \leftrightarrow e\right\rangle$ & $15:\left\langle L_{1} \rightarrow F_{3} ; \underline{l_{3}} \leftrightarrow e\right\rangle$ & $21:\left\langle a_{r} \rightarrow e ; L_{1}^{\prime \prime} \leftrightarrow L_{1}\right\rangle$ \\
\hline $\begin{array}{l}10:\left\langle l_{1}^{\prime \prime \prime \prime} \rightarrow \overline{l_{1}} ; e \leftrightarrow e\right\rangle \\
11:\left\langle\overline{l_{1}} \rightarrow \overline{\overline{l_{1}}} ; e \leftrightarrow e\right\rangle\end{array}$ & $\begin{array}{l}16:\left\langle e \rightarrow \underline{\underline{l_{3}}} ; F_{3} \leftrightarrow \underline{l_{3}}\right\rangle \\
17:\left\langle\underline{l_{3}} \rightarrow \underline{l_{3}} ; \underline{\underline{l_{3}}} \leftrightarrow e\right\rangle\end{array}$ & $\begin{array}{l}22:\left\langle L_{1} \rightarrow e ; e \leftrightarrow e\right\rangle \\
23:\left\langle l_{1}^{\prime \prime} \rightarrow e ; L_{1}^{\prime} \leftrightarrow F_{3}\right\rangle \\
24:\left\langle F_{3} \rightarrow e ; e \leftrightarrow e\right\rangle\end{array}$ \\
\hline
\end{tabular}

At the first phase of the simulation of the $S U B$ instruction the first agent generates object $l_{1}^{\prime}$, which is consumed by the second agent. The agent $B_{2}$ generates symbol $L_{1}$ and tries to consume one copy of symbol $a_{r}$. If there is any $a_{r}$, the agent sends to the environment object $L_{1}^{\prime \prime}$ and consumes $L_{1}$. After this step the first agent consumes $L_{1}^{\prime \prime}$ or $L_{1}$ and rewrites it to $l_{2}$ or $l_{3}$. The objects $\underline{x}, \bar{x}$ and $\overline{\bar{x}}$ are used for a synchronization of the computation in both agents and for storing information about the state of the computation.

Instruction $l_{1}:\left(S U B(r), l_{2}, l_{3}\right)$ is simulated by the following sequence of steps.

If the register $r$ stores value zero : If the register $r$ stores nonzero value:

\begin{tabular}{|c|c|c|c|c|c|c|c|c|c|c|c|}
\hline & & igurat & ion of $\Pi$ & & & & conf & surati & of $\Pi$ & & \\
\hline & $B_{1}$ & $B_{2}$ & $E n v$ & $P_{1}$ & $P_{2}$ & & $B_{1}$ & $B_{2}$ & Env & $P_{1}$ & $P_{2}$ \\
\hline 1. & $l_{1} e$ & $e e$ & $a_{r}^{x}$ & 6 & - & 1. & $l_{1} e$ & $e e$ & & 6 & - \\
\hline 2 . & $l_{1}^{\prime} e$ & $e e$ & $a_{r}^{x}$ & 7 & - & 2 . & $l_{1}^{\prime} e$ & $e e$ & & 7 & - \\
\hline 3 . & $l_{1}^{\prime \prime} e$ & $e e$ & $l_{1}^{\prime} a_{r}^{x}$ & 8 & 18 & 3. & $l_{1}^{\prime \prime} e$ & $e e$ & $l_{1}^{\prime}$ & 8 & 18 \\
\hline 4. & $l_{1}^{\prime \prime \prime} e$ & $L_{1} l_{1}^{\prime}$ & $l_{1}^{\prime \prime} a_{r}^{x}$ & 9 & 19 & 4. & $l_{1}^{\prime \prime \prime} e$ & $L_{1} l_{1}^{\prime}$ & $l_{1}^{\prime \prime}$ & 9 & 19 \\
\hline 5 . & $l_{1}^{\prime \prime \prime \prime} e$ & $L_{1}^{\prime} l_{1}^{\prime \prime}$ & $L_{1} a_{r}^{x}$ & 10 & 20 & 5. & $l_{1}^{\prime \prime \prime \prime} e$ & $L_{1}^{\prime} l_{1}^{\prime \prime}$ & $L_{1}$ & 10 & \\
\hline 6 . & $\overline{l_{1}} e$ & $L_{1}^{\prime \prime} a_{r}$ & $L_{1} L_{1}^{\prime} a_{r}^{x-1}$ & 11 & 21 & 6. & $\overline{l_{1}} e$ & $L_{1}^{\prime} l_{1}^{\prime \prime}$ & $L_{1}$ & 11 & \\
\hline 7 . & $\overline{\overline{l_{1}}} e$ & $e L_{1}$ & $L_{1}^{\prime \prime} a_{r}^{x-1}$ & 12 & 22 & 7. & $\overline{\overline{l_{1}}} e$ & $L_{1}^{\prime} l_{1}^{\prime \prime}$ & $L_{1}$ & 13 & \\
\hline 8. & $\underline{l_{2}} L_{1}^{\prime \prime}$ & $e e$ & $a_{r}^{x-1}$ & 14 & - & 8. & $\underline{l_{3}} L_{1}$ & $L_{1}^{\prime} l_{1}^{\prime \prime}$ & & 15 & - \\
\hline 9 . & $l_{2} e$ & & $a_{r}^{x-1} \underline{l}_{2}$ & & & 9 . & $F_{3} e$ & $L_{1}^{\prime} l_{1}^{\prime \prime}$ & $\underline{l_{3}}$ & 16 & - \\
\hline & & & & & & 10 . & $\underline{l_{3} l_{3}}$ & $L_{1}^{\prime} l_{1}^{\prime \prime}$ & $F_{3}$ & 17 & 23 \\
\hline & & & & & & 11. & $\overline{l_{3}} e$ & $F_{3} e$ & $\underline{\underline{l_{3}}} L_{1}^{\prime}$ & 2 or 6 & 24 \\
\hline & & & & & & 12 . & $? ?$ & $e e$ & $\underline{\underline{l_{3}}} L_{1}^{\prime}$ & & \\
\hline
\end{tabular}

(4) For halting instruction $l_{h}$ no program is added to the sets $P_{1}$ and $P_{2}$.

$\mathrm{P}$ colony $\Pi$ correctly simulates all computations of the register machine $M$ and the number contained on the first register of $M$ corresponds to the number of copies of the object $a_{1}$ present in the environment of $\Pi$. 


\section{Conclusions}

We have shown that the $\mathrm{P}$ colonies with capacity $k=2$ and without checking programs with height at most 2 are computationally complete. In Section 3 we have shown that the $\mathrm{P}$ colonies with capacity $k=1$ and with checking/evolution programs and 4 agents are computationally complete.

We have verified also that partially blind register machines can be simulated by $\mathrm{P}$ colonies with capacity $k=1$ without checking programs with two agents. The generative power of $N P C O L_{p a r} K(1, n, *)$ for $n=2,3$ remains open.

In Section 4 we have studied P colonies with capacity $k=2$ without checking programs. Two agents guarantee the computational completeness in this case.

Remark 1. This work has been supported by the Grant Agency of Czech Republic grants No. 201/06/0567 and by IGS SU 32/2007.

\section{References}

1. Ciencialová, L., Cienciala, L.: Variations on the theme: P Colonies, Proceedings of the $1^{\text {st }}$ International workshop WFM'06 (Koláŕ, D., Meduna, A., eds.), Ostrava, 2006, pp. $27-34$.

2. Csuhaj-Varjú, E., Kelemen, J., Kelemenová, A., Păun, Gh., Vaszil, G.: Cells in environment: P colonies, Multiple-valued Logic and Soft Computing, 12, 3-4, 2006, pp. 201-215.

3. Csuhaj-Varjú, E., Margenstern, M., Vaszil, G.: P Colonies with a bounded number of cells and programs. Pre-Proceedings of the $7^{\text {th }}$ Workshop on Membrane Computing (H. J. Hoogeboom, Gh. Păun, G. Rozenberg, eds.), Leiden, The Netherlands, 2006, pp. 311-322.

4. Freund, R., Oswald, M.: P colonies working in the maximally parallel and in the sequential mode. Pre-Proceedings of the $1^{\text {st }}$ International Workshop on Theory and Application of P Systems (G. Ciobanu, Gh. Păun, eds.), Timisoara, Romania, 2005, pp. $49-56$.

5. Greibach, S. A.: Remarks on blind and partially blind one-way multicounter machines. Theoretical Computer Science, 7(1), 1978, pp. 311-324.

6. Kelemen, J., Kelemenová, A.: On P colonies, a biochemically inspired model of computation. Proc. of the $6^{\text {th }}$ International Symposium of Hungarian Researchers on Computational Intelligence, Budapest TECH, Hungary, 2005, pp. 40-56.

7. Kelemen, J., Kelemenová, A., Păun, Gh.: Preview of $P$ colonies: A biochemically inspired computing model. Workshop and Tutorial Proceedings, Ninth International Conference on the Simulation and Synthesis of Living Systems, ALIFE IX (M. Bedau at al., eds.) Boston, Mass., 2004, pp. 82-86.

8. Minsky, M. L.: Computation: Finite and Infinite Machines. Prentice Hall, Engle-wood Cliffs, NJ, 1967.

9. Păun, Gh.: Computing with membranes. Journal of Computer and System Sciences 61, 2000, pp. 108-143.

10. Păun, Gh.: Membrane computing: An introduction. Springer-Verlag, Berlin, 2002.

11. P systems web page: http://psystems.disco.unimib.it 
